\title{
EQUIPAMENTO EXPERIMENTAL PARA DETERMINAÇÃO DE ISOTERMAS DE ADSORÇÃO PELO MÉTODO DINÂMICO
}

\author{
PRADO FILHO, L.G.; SABBAGH, $\mathbf{M}^{1}$. \\ Departamento de Ciência e Tecnologia Agroindustrial - ESALQ/USP - C.P. 9 - CEP:13418-900 - Piracicaba - SP.
}

\begin{abstract}
RESUMO: Foi realizada a avaliação do desempenho de equipamento experimental para a determinação de curvas de umidade de equilibrio - isoterma de adsorção - de farinhas pelo método dinâmico. Para o ajuste dos valores definidos $(0,51 ; 0,57 ; 0,67 ; 0,75 ; 0,79$ e 0,81$)$ de atividade de água - Aa - na corrente de ar, foram usadas soluçōes saturadas de sais. A comparação da eficiência do equipamento, com e sem recirculação de ar, foi feita determinando-se a isoterma de adsorção de farinha de soja a $35^{\circ} \mathrm{C}$. A versão com recirculação de ar permite a obtenção de dados de equilibrio em elevados níveis de Aa, nos quais há perda das amostras por crescimento microbiano quando se emprega a versāo sem recirculação. Os tempos necessários para a obtenção do equilibrio da farinha com as umidades relativas das correntes de ar são cerca de $50 \%$ menores na versão com recirculação de ar. Descritores: isoterma de adsorção, atividade de água, farinha de soja.
\end{abstract}

\section{EXPERIMENTAL APPARATUS FOR THE DETERMINATION OF SORPTION ISOTIIERMS BY THE DYNAMIC METIIOD.}

\begin{abstract}
ARSTRACT: The evaluation of the performance of an experimental apparatus for the determination of flour sorption isotherms was carried out. To adjust the water activity - $\mathrm{Aa}$ - values $(0.51 ; 0.57 ; 0.67 ; 0.75 ; 0.79$ and 0.81$)$ of the air stream, saturated salt solutions were used. The comparison of the apparatus with and without air recycling, was done by determining the sorption isotherm of soybean flour at $35^{\circ} \mathrm{C}$. The air recycling model enables to obtain reliable data in a high Aa range, where samples are usually impaired by microbial growth when the other model is used. Time to obtain equilibrium for soybean flour with the air recycling model was about $50 \%$ less than for the model without air recycling.
\end{abstract}

Key Words: sorption isotherm, water activity, soybean flour.

\section{INTRODUÇÃO}

Conhecendo-se a curva de umidade de equilibrio - isoterma de adsorção - de um alimento ou matéria prima, é possível gerenciar-se os processos de desidratação, embalagem e armazenagem de modo a obter-se, nos mesmos, níveis de atividade de água que minimizem as alterações indesejáveis durante a armazenagem (MOSSEL, 1975; LABUZA, 1980).

O presente trabalho descreve a montagem de equipamento experimental simples para a determinação de isotermas de adsorção de farinhas, em espaços de tempo mais curtos que aqueles necessários quando se trabalha com métodos de equilibrio estático em dessecadores. Isto possibilita o trabalho com valores mais altos de atividade de água - Aa - sem que ocorram perdas de amostras devidas a crescimento fúngico.

O princípio básico seguido na concepção do equipamento foi o de ajuste da Aa desejada na corrente de ar através do borbulhamento deste em soluções saturadas de sais.

Foram testadas duas versões do equipamento proposto, uma sem recirculação de ar e outra com recirculação. Os desempenhos de ambas as versões foram comparados usando-se farinha de soja como material de estudo, determinando-se os tempos necessários para o estabelecimento de equilibrio com correntes de ar de Aa definidas e, construindo-se as isotermas parciais de adsorção a partir dos dados de equilibrio obtidos a $35^{\circ} \mathrm{C}$.

\footnotetext{
${ }^{1}$ Bolsista da FAPESP.
} 


\section{MATERIAIS E MÉTODOS}

Para obtenção da farinha de soja foram empregados grãos da variedade Santa Rosa, fornecidos pelo IAC-Campinas,SP, seguindo-se o esquema proposto por KELLOR (1974). A farinha foi então tratada por vapor fluente para inativação de fatores antinutricionais sendo em seguida eliminado o excesso de umidade a $40^{\circ} \mathrm{C}$ por 6 horas. Esta farinha desengordurada e seca, sem controle da umidade final e preparada em várias bateladas, foi usada para os experimentos, após passagem por peneira 20 "mesh".

As soluções salinas saturadas (em presença do sal em excesso), utilizadas para ajustar as Aa definidas na corrente de ar nos experimentos de equilibrio das amostras de farinha de soja, são as mencionadas por SMITH (1971) resumidas na Figura 1 apenas para os valores de umidades relativas estudados neste trabalho.

$O$ equipamento usado para a determinação da umidade relativa de equilíbrio, na versão sem recirculação de ar, baseada no modelo proposto por LONCIN et al. (1968), consta de um regulador de pressão, com manômetro e filtro de água e óleo acoplados, marca DEVILBISS modelo P-HLE5010 , três garrafas lavadoras de gás e uma câmara de equilíbrio. A primeira garrafa contém água destilada, a segunda, uma solução saturada do sal pertinente, e a terceira, acomoda lã de vidro como filtro de eventuais gotículas da solução arrastadas pela corrente de ar.

\begin{tabular}{|lcr|}
\hline \multicolumn{1}{|c|}{ Sal } & $\begin{array}{c}\text { Umidade } \\
\text { relativa \% }\end{array}$ & $\begin{array}{r}\text { Atividade } \\
\text { de água }\end{array}$ \\
\hline Nitrato de Magnésio & 51 & 0,51 \\
Brometo de Sódio & 57 & 0,57 \\
Cloreto de Cobre & 67 & 0,67 \\
Cloreto de Sódio & 75 & 0,75 \\
Sulfato de Amôneo & 79 & 0,79 \\
Brometo de Potássio & 81 & 0,81 \\
\hline
\end{tabular}

Figura 1 - Umidades relativas e atividades de água de soluções salinas saturadas a $35^{\circ} \mathrm{C}$. Adaptada de SMITH (1971).

A câmara de equilibrio é construída de tubo de PVC com $100 \mathrm{~mm}$ diâmetro externo e 300 $\mathrm{mm}$ de altura, cujas extremidades são fechadas por tampas do mesmo material, sendo a superior remo- vível e a inferior colada. Tais materiais são usuais em hidráulica de construções civis. A entrada e a saída do ar na câmara são de tubos de vidro $(7 \mathrm{~mm}$ de diâmetro externo) colados com resina epóxi.

Dentro da câmara é ajustado, com folga para facilitar a remoção, um suporte construido com três varões de latão ( $3 \mathrm{~mm}$ de diâmetro e 270 $\mathrm{mm}$ de comprimento) aos quais estão colados cinco discos perfurados de chapa de PVC $(1,5 \mathrm{~mm}$ de espessura). Os três discos intermediários são usados como suporte das placas de Petri contendo as amostras de farinha, em triplicata. As conexões entre os elementos do equipamento são feitas por tubos de látex. A Figura 2 mostra o esquema de montagem dessa versão do equipamento.

$\mathrm{Na}$ versão com recirculação de ar, uma adaptação simplificada do modelo proposto por GAL (1975), é usada apenas uma garrafa lavadora de gás com solução salina saturada, ao gargalo da qual se adapta lã de vidro para filtração de gotículas. Uma bomba de diafragma com vazão média de $600 \mathrm{ml} /$ minuto (desacoplada do equipamento), acomodada em recipiente de PVC de dois litros de capacidade, impulsiona e aspira o ar em circuito fechado. A câmara de equilibrio, o suporte e as ligações são idênticos aos da versão sem recirculação. A Figura 3 mostra o esquema de montagem desta versão do equipamento.

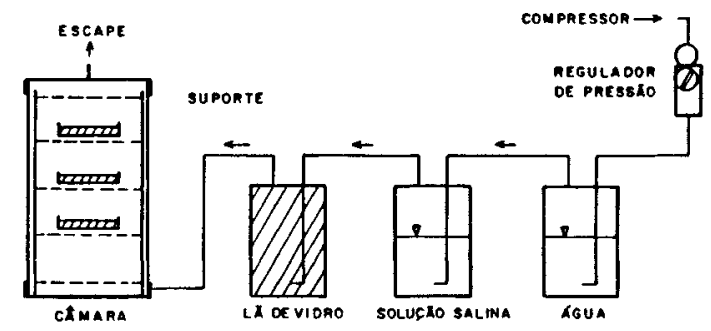

Figura 2 - Equipamento sem recirculação de ar.

Para as determinações das umidades de equilíbrio da farinha de soja com a versão sem circulação de ar foi adotado o seguinte procedimento. Três placas de Petri, de $60 \mathrm{~mm}$ de diâmetro, secas em estufa e equilibradas por duas horas em dessecador com a solução salina do experimento a realizar, receberam quatro gramas 
TABELA 1 - Teores finais de umidade em $\mathrm{g} \mathrm{H}_{2} \mathrm{O} / 100 \mathrm{~g}$ MS obtidos para farinha de soja (média de três amostras).

\begin{tabular}{lcccccc}
\hline \hline & \multicolumn{7}{c}{ Atividade de água } \\
\cline { 2 - 7 } Equipamento & 0,51 & 0,57 & 0,67 & 0,75 & 0,79 & 0,81 \\
\hline Sem recirculação & 6,36 & 9,38 & 11,57 & nd & nd & nd \\
Com recirculação & 6,28 & 9,34 & 11,55 & 14,70 & 19,48 & 21,77 \\
\hline \hline
\end{tabular}

nd = mela das amostras e/ou crescimento fúngico visível

(aproximação de centigrama) cada uma da farinha, sendo então posicionadas no suporte e este introduzido na câmara de equilíbrio. Fechada a câmara, ligou-se o compressor ajustando a pressão para $1,6 \mathrm{Kgf} / \mathrm{cm}^{2}$, o que resulta em uma vazão média de $1200 \mathrm{ml}$ de ar/min na saída do equipamento. Todo o conjunto é mantido em estufa a $35^{\circ} \mathrm{C}$ com variação máxima de $1^{\circ} \mathrm{C}$.

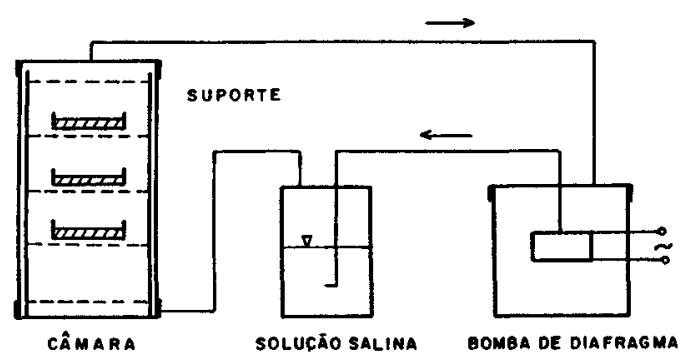

Figura 3 - Equipamento com recirculação de ar.

Com intervalos de 12 horas as amostras foram pesadas considerando-se terminado $o$ experimento quando não havia mais variação da primeira casa decimal da média do peso das três amostras, por três pesagens consecutivas. Nas operações de pesagem apenas as placas com as amostras são retiradas da estufa e transferidas para dessecador com solução salina pertinente, sendo retiradas deste ambiente apenas no momento da aferição do peso. A cada pesagem a posição das placas na camara foi modificada, para uniformizar a exposição das mesmas à corrente de ar. Depois de alcançado o equilibrio, as amostras são secas a vácuo $\left(80 \mathrm{~mm} \mathrm{Hg}\right.$ de pressão residual) a $60^{\circ} \mathrm{C}$ por 6 horas, sendo então determinado o peso seco e calculada a umidade absoluta em gramas (aproximação de centigrama) de água por 100 gramas de matéria seca nas amostras, em cada pesada executada durante o experimento.

Para as determinações com a versão com recirculação de ar, o procedimento é idêntico ao anteriormente descrito, porém, $o$ intervalo entre as pesadas foi de apenas 6 horas. Os gráficos de equilíbrio e as isotermas de adsorção apresentados são resultantes do processamento, pelo programa ENERGRAPH da Enertronics Research Inc, dos dados experimentais obtidos.

\section{RESULTADOS E DISCUSSÃO}

As Figuras 4 e 5 mostram claramente a superioridade do equipamento com ar recirculado sobre o de ar não recirculado. Nas $\mathrm{Aa}$ 0,51, 0,57 e 0,67 os tempos necessários para o equilíbrio no sistema com recirculação são inferiores à metade daqueles exigidos pelo equilibrio no sistema sem recirculação. Em valores maiores de Aa não houve termos de comparação uma vez que as amostras perdiam-se por crescimento microbiano evidenciado pela mela da farinha (atividade amilolítica) ou crescimento visível de micélio fúngico.

Aplicando-se o teste $T$ (Student) proposto por STEEL \& TORRIE (1960), não foi detectada diferença significativa entre os resultados obtidos com ambas as versões (TABELA 1), para um erro experimental calculado de $0,98 \%$. Este pequeno erro experimental mostra que considerar a estabilidade da primeira casa decimal da média dos 
pesos das três amostras como critério para o término dos experimentos, oferece precisão satisfatória para o tipo de estudo realizado.

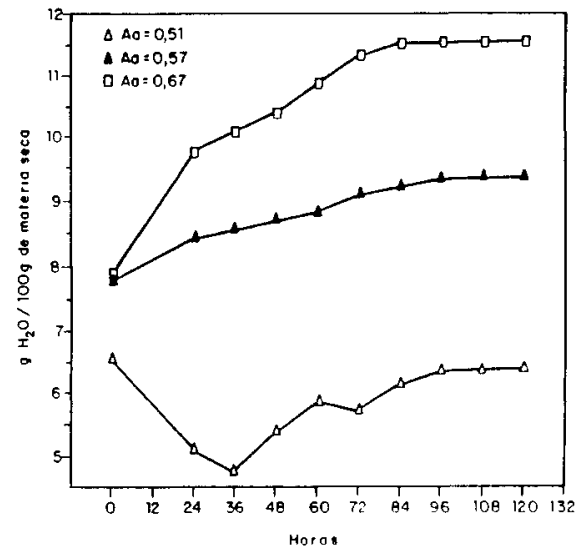

Figura 4 - Tempos em horas para o equilibrio da farinha de soja no equipamento sem recirculação de ar.

Sem dúvida a recirculação propicia o estabelecimento rápido de uma umidade relativa estável na corrente de ar (GUR-ARIEH et al., 1965) o que se reflete na rapidez com que as amostras atingem o equilíbrio. Mesmo com tempos sensivelmente menores para atingir o equilbrio, o sistema com recirculação de ar dispensa a tomada de dados, no mínimo, nas primeiras 24 horas de experimentação, como pode ser constatado na Figura 5. Isto representa uma economia apreciável de trabalho.

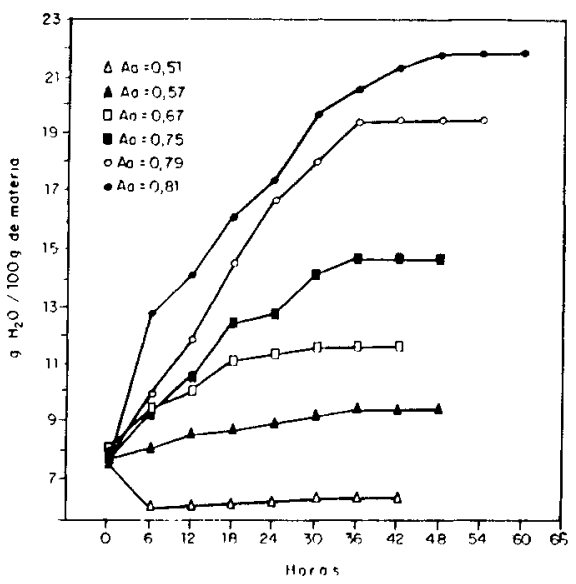

Figura 5 - Tempos em horas para o equilibrio da farinha de soja no equipamento com recirculação de ar.

Sci. Agric., Piracicaba, 50(3):455-459, out./dez., 1993
Como consequência do desempenho superior da versão com recirculação de ar, a isoterma de adsorção construida a partir dos dados obtidos neste sistema é bem mais completa do que aquela obtida sem recirculação (Figuras 6 e 7), abrangendo todo o âmbito de atividades de água que tem interesse prático no estudo de estocagem e vida de prateleira de farináceos e similares.

Outro ponto evidenciado pelo presente estudo é o baixo custo da versão mais eficiente do equipamento pois, uma bomba de diafragma, mesmo de vazão superior a da empregada nos ensaios pode ser adquirida por uma fração do custo de um pequeno compressor e respectivos regulador e filtro.

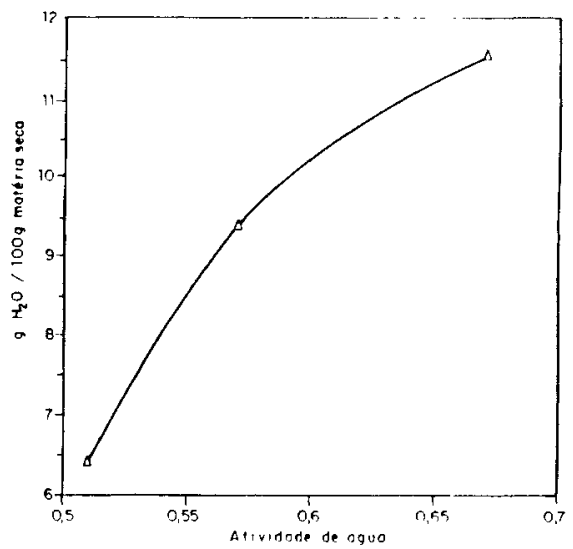

Figura 6 - Isoterma parcial de adsorção de farinha de soja. Dados de equilibrio obtidos sem recirculação de ar.

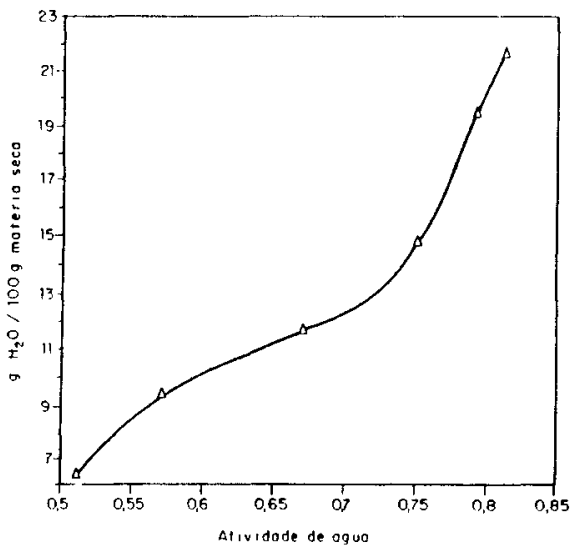

Figura 7 - lsoterma parcial de adsorção de farinha de soja. Dados de equilibrio obtidos com recirculação de ar. 


\section{CONCLUSÕES}

O equipamento proposto na versão com recirculação de ar, de custo de construção mais baixo, mostrou-se bastante eficiente para a obtenção de dados de equilibrio a partir dos quais foi construída a isoterma parcial de adsorção da farinha de soja, podendo seu uso ser extendido para outros materiais particulados de estrutura similar.

Os tempos necessários para a farinha de soja atingir o equilibrio, com as correntes de ar com as atividades de água estudadas, foram sensivelmente mais curtos que aqueles que seriam necessários com o emprego de método estático de equilíbrio. Isto tornou possível trabalhar com valores de atividade de água mais elevados sem que houvesse perdas devidas a crescimento microbiano, e obter uma isoterma parcial de absorção mais completa, abrangendo todo o âmbito de atividades de água de interesse prático em armazenagem.

Nas condições em que foram realizados os experimentos com recirculação de ar, ficou demonstrado não ser necessária a tomada de dados antes de 24 horas após o início dos mesmos, o que importa em uma economia apreciável de mão de obra.

\section{REFERÊNCIAS BIBLIOGRÁFICAS}

GAL, S. Recent advances in techniques for the determination of sorption isothermes. In: DUCKWORTH, R.B. (Ed). Relations of foods. London: Academic Press, 1975, p.139-154.
GUR-ARIEH, B.E.; NELSON, A.I.; STEINBERG, M.P. \& WEI, L.S. A method for rapid determination of moisture-adsorption isotherms of solid particles. Joumal of Food Science. Chicago,v.30, n.1, p.105$110,1965$.

KELLOR, R.L. Defatted soy flour and grits. Journal of the American Oil Chemist's Society, Chicago, v.51, n.1, p.77-80, 1974.

LABUZA, T.P. The effect of water activity on reaction kinetics of food deterioration. Food Technology, Chicago, v.36, n.4, p.92-97, 1980.

LONCIN, M.; BIMBENET, J.J.; LENGES, J. Influence of the activity of water on the spoilage of foodstuffs. Journal of Food Technology, Oxford, v.3, p.131-142, 1968 .

MOSSEL, D.A.A. Water and micro-organisms in foods: a synthesis. In: DUCKWORTH, R.B. (Ed.). Water relations of foods. London: Academic Press, 1975. p.347-361.

SMITH, P.R. The determination of equilibrium humidity or water activity in foods. A literature review. Scientific and Technical Surveys - British Food Manufacturing Industries Research Association, Leatherhead, n.70, p.2-26, March, 1971

STEEL, R.G.O.; TORRIE, J.H. Principles and procedures of statistics. New York, Mac Grow-Hill, 1960. 481 p.

Enviado para publicação em 12.05 .91

Aceito para publicação em 01.06 .93 